\title{
Falls in Geriatric Population- Now to Future!
}

\author{
Pooja Attrey* and Mukesh Sharma \\ Assistant Professor, TDTR DAV Institute Of Physiotherapy \& Rehabilitation, India
}

*Corresponding author: Pooja Attrey, Assistant Professor, TDTR DAV Institute of Physiotherapy \& Rehabilitation, Professor Colony, Yamunanagar-135 001, Haryana, India

Submission: 㘹 March 14, 2018; Published: 眥 March 16, 2018

\section{Opinion}

Falls, being the leading cause of injury deaths and disabilities, are quite common in the geriatric population, more often above 65 years of age. Every 32 minutes, an elderly person turns up at a public hospital emergency department because of an injury from a fall. A problem that get worsen with ageing, fall also brings in its wake higher healthcare costs and lowers the quality of life for those affected.

Among 1 of 3 older adult falls, that occurs yearly, 24\% sustains fatal injuries while $6 \%$ results in fractures. In late 90 's, nearly 9,000 persons aged $>65$ years died from falls. Among the reported cases, $20 \%-30 \%$ sustain moderate to severe injuries that reduce mobility \& independence and increase the risk for premature death.

Between both genders, women are 3 times more likely to require medical attention for fall related injuries. If reviewed among different age groups, older adults are 5 times more likely to end up in hospital because of fall injuries. Fall-related injuries among older adults results mostly in fractures of the hip>spine>upper arm> forearm and lastly bones of the pelvis, hand, and ankle. Out of all, Hip fracture is a leading cause of morbidity and excess mortality among older adults. All these injuries impose additional cost on health care systems. The cost of falls in the US health care system alone is expected to increase to $\$ 54.9$ billion by 2020 [1].

\section{Now to understand what a fall is, let's go further}

A fall can be defined as a sudden, unintentional change in position causing an individual to land at a lower level, on an object, the floor, or the ground, other than as a consequence of sudden onset of paralysis, epileptic seizure, or overwhelming external force .It is an event caused by multifactorial aetiology like age associated diseases (Parkinson's disease), cognitive decline and musculoskeletal problems in association with certain physiological changes like impairment of sensory system, righting reflex and decrease in lean body mass. The physiological changes might just be the 'normal' age related changes.

\section{Causes of Fall in Older Persons}

The causes of fall can be Extrinsic or Intrinsic. Falls due to intrinsic causes like visual, cognitive, vestibular, neurological, musculoskeletal problems and drug induced recurrent falls are common in people $>70$ years. Among these causes gait \& balance disorders or weakness is main accounting for $17 \%$ of cases. On the other hand, Dizziness and vertigo are reasons for 13\% and Drop attack, Confusion, Postural hypotension accounts for 9\%, 5\% and $3 \%$ of cases respectively. Visual disorder is causes for fall in $2 \%$ of reported cases while Syncope in $0.3 \%$ cases. Extrinsic factors like accident \& environment-related causes results in $31 \%$ of total reported cases of elderly falls [2].

\section{Screening \& Assessment of Falls}

The subject must be asked about the history of fall every year, if the annual frequency of falls is increased due to gait \& balance disturbance, the subject is required to go for assessment including Gait, Posture and Balance. Timed up \& Go Test is a simple tool to assess fall risk \& balance. It is a quick and easy test requiring a few minutes only. The subject start by sitting back in a chair with armrests and is timed while rising from the chair, walking three meters then turns around [3], walking back to the chair \& sitting back down. Postural stability, gait, stride length, sway, and step length should be observed. The normal value is 10 seconds or less but Kaiser Permanente uses the operational value of 14 seconds or less.

\section{Interventions and Prevention Programs}

The best international reviews advocates multifaceted programs, including counselling \& health education awareness, exercise, vision correction, medication review and adjustment, and environmental modification. Muscle strengthening and balance retraining programmes or Tai Chi (which combines strength and balance training) may be effective as sole interventions for preventing falls in elderly [4].

\section{Falls \& Injury Prevention Policies}

To reduce frequency of fall injuries among older persons, holistic approach of management should be supported, adapted and implemented. Governments must be petitioned to make injury prevention policy on priority on the agenda of health issues [5]. Increase in Funding As well as Awareness on existing policies is required. Health care providers and family support system (if any) must work together to reduce falls. Also it would be beneficial even for novice or new care taker to understand the situation of 
the elderly if risk indicators will be included in a subject's medical records. The communication between injury prevention groups must be increased to have updated information on the status of the subject.

There is still a need to increase research in the field ofgerontology. Evidence-based practices must be incorporated in management. To improve the effectiveness of treatment and rehabilitation of falls injury along with the risk factors monitoring and implementation of interventions to prevent fall injuries, research to create more evidence needs to be supported [6]. A systematic pro-active, multifactorial approach to fall risk assessment and injury prevention is essential. Better fall screening tools and other assessment procedures needs to be designed \& validated. Fall Preventive Programme including life style changes and active participation in regular exercise programmes is to be strictly followed after middle age for every community dwelling adult.

Also awareness education about the benefits of regular exercises must be given to older persons. Along with all other essentials, The core medical curricula must include injury prevention rather just cure. Though there is a definite need of the long-term fall injury prevention strategy that incorporates risk assessments as well as intervention strategies, we as a heath care system \& society still need to incorporate much more of our extended health management learning into practical implementation for our geriatric population.

\section{References}

1. Aimee Lee, Kuo Wei Lee, Peter Khang (2013) Preventing Falls in the Geriatric Population. Perm J 17(4): 37-39.

2. Feder G, Cryer C, Donovan S, Carter Y (2000) Guidelines for the prevention of falls in people over 65. The Guidelines' Development Group. BMJ 321(7267): 1007-1011.

3. Chang JT, Morton SC, Rubenstein LZ, Mojica WA, Maglione M, et al. (2004) Interventions for the prevention of falls in older adults: systematic review and meta-analysis of randomised clinical trials. BMJ 328(7441): 680.

4. Leslie Kernisan: Why Older People Fall \& How to Reduce Fall Risk.

5. Injury prevention \& control: data \& statistics (WISQARS).

6. Costs of falls among older adults.
Creative Commons Attribution 4.0

International License

For possible submissions Click Here
Submit Article

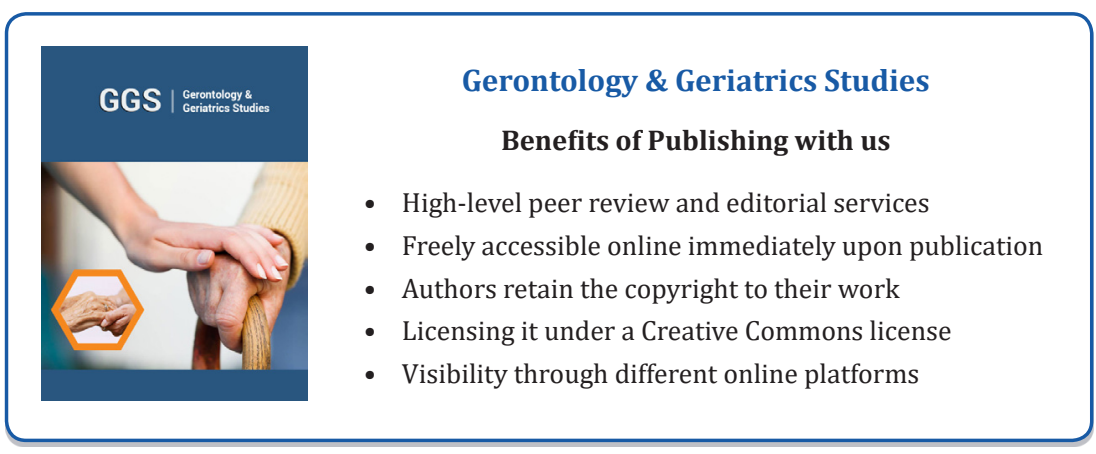

